\title{
A SOM based Anomaly Detection Method for Wind Turbines Health Management through SCADA Data
}

\author{
Mian Du ${ }^{1,2,3}$, Lina Bertling Tjernberg ${ }^{3}$, Shicong $\mathrm{Ma}^{1}$, Qing $\mathrm{He}^{1}$, Lin Cheng ${ }^{2}$ and Jianbo Guo ${ }^{1}$ \\ ${ }^{1}$ China Electric Power Research Institute, Beijing, Haidian, 100192, China \\ dm13@mails.tsinghua.edu.cn \\ ${ }^{2}$ Department of Electrical Engineering, Tsinghua University, Beijing, Haidian, 100084, China \\ ${ }^{3}$ KTH Royal Institute of Technology, Stockholm, Sweden
}

\begin{abstract}
In this paper, a data driven method for Wind Turbine system level anomaly detection and root sub-component identification is proposed. Supervisory control and data acquisition system (SCADA) data of WT is adopted and several parameters are selected based on physical knowledge in this domain and correlation coefficient analysis to build a normal behavior model. This model which is based on Self-organizing map (SOM) projects higher-dimensional SCADA data into a two-dimension-map. Afterwards, the Euclidean distance based indicator for system level anomalies is defined and a filter is created to screen out suspicious data points based on quantile function. Moreover, a failure data pattern based criterion is created for anomaly detection from system level. In order to track which sub-component should be responsible for an anomaly, a contribution proportion (CP) index is proposed. The method is tested with a two-month SCADA dataset with the measurement interval as 20 seconds. Results demonstrate capability and efficiency of the proposed method.
\end{abstract}

\section{INTRODUCTION}

Wind energy is considered an effective way to relieve the carbon dioxide risk caused by consuming traditional fossil resources. According to the statistics on wind energy published by the European wind energy association in Feb. 2016 (European Wind Energy Association, 2016), until $2015,142 \mathrm{GW}$ of wind energy in total has been installed in Europe and $11 \mathrm{GW}$ of it includes offshore. Moreover, what should be considered as milestone is that in 2015, wind energy has substituted hydro as the third largest power source in the European Union (EU) with $15.6 \%$ share of

Mian Du et al. This is an open-access article distributed under the terms of the Creative Commons Attribution 3.0 United States License, which permits unrestricted use, distribution, and reproduction in any medium, provided the original author and source are credited. total power capacity. Figure 1 shows the annual installation of both onshore and offshore wind energy in EU.

Wind turbines are capital-intensive equipment compared to conventional fossil resource based technologies such as natural gas power generators, where as much as $25-30 \%$ of costs are related to operations and maintenance (O\&M) (Milborrow, 2006). Due to the huge amount of O\&M cost of a wind farm, keeping WTs working efficiently and formulating cost effective maintenance schedules are the main interests of shareholders, especially in offshore wind farms.

While realizing the objective of decreasing the O\&M cost needs various information, one of the most significant part is the health condition of wind turbines. In recently published papers (de Azevedo, Araújo, \& Bouchonneau, 2016) and (Kandukuri, Karimi, \& Robbersmyr, 2016), related excellent works have been introduced. Based on adopted approaches, research on wind turbine health condition monitoring can generally be classified as two

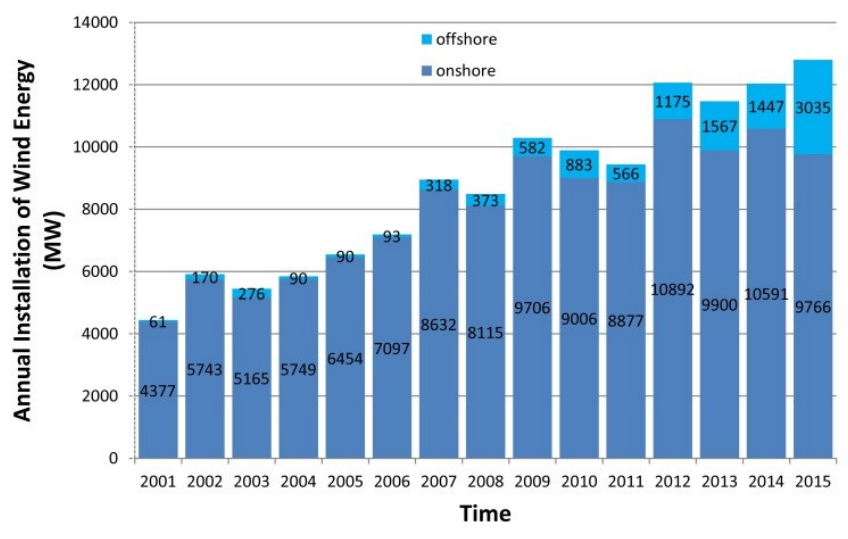

Figure 1. Annual onshore and offshore wind energy installation (MW) (European Wind Energy Association, 2016) 
main branches, data driven approach (Sun, Li, Wang, \& Lei, 2016) and analytical approach (Bruce, Long, \& DwyerJoyce, 2015).

While a physic based analytical model is easier for understanding and interpretation, the nonlinear relationship among the subcomponents of a wind turbine make it difficult to model a wind turbine from a system level (de Bessa, Palhares, D'Angelo, \& Chaves Filho). Even though in (Breteler, Kaidis, Tinga, \& Loendersloot, 2015) a system level physics based model is proposed for failure prognosis, it still keeps the assumption that each component works independently. Moreover, the component failure detection in that paper is still based on data from condition monitoring system (CMS) and supervisory control and data acquisition system (SCADA). As access to wind turbine O\&M data is much easier with the development of advanced sensor technology, system level methods for wind turbine health management are more preferable from O\&M perspective. Data driven approach from a system level perception is attracting more interest in research field (de Bessa, Palhares, D'Angelo, \& Chaves Filho) (Sun, Li, Wang, \& Lei, 2016) (de Andrade Vieira \& Sanz-Bobi, 2013) (Marhadi \& Skrimpas, 2015).

Considering the data type adopted in this field, vibration data (Yang, et al., 2016), acoustic data (Park, Sohn, Malinowski, \& Ostachowicz, 2016) and SCADA data (Schlechtingen, Santos, \& Achiche, 2013) are most widely used in recent published papers. Comparing with the previous two data resources, SCADA data has comprehensive information of nearly all subcomponents and is pointed out to be the most economic data resource for developing a CMS for wind turbines (Xiang, Watson, \& Liu, 2009). Besides, there is a large amount of SCADA data available which contains both wind turbine operation status and measurements of signals as temperature, pressure, voltage and current. From a practical point of view, a SCADA system is constituted by many sensors distributed to each subcomponent. For some key components as generator, gear box and rotor system, several sensors are equipped to get more thorough information (Sun, Li, Wang, \& Lei, 2016). Therefore, monitoring operation status and health condition through SCADA data is more cost-effective.

Considering various data-driven methods, such as neural network (NN) (de Andrade Vieira \& Sanz-Bobi, 2013), fuzzy based approach (Sun, Li, Wang, \& Lei, 2016), support vector machine (SVM) (Santos, Villa, Reñones, Bustillo, \& Maudes, 2015), and Bayesian network based approach (Schlechtingen, Santos, \& Achiche, 2013) have been used to model wind turbine behavior with SCADA data. Although each approach has advantages and limitations, great findings have been provided by the previous papers. In (Santos, Villa, Reñones, Bustillo, \& Maudes, 2015), a SVM based solution is created for failure detection with classification of operational states of a wind turbine. While in (Castellani,
Astolfi, Sdringola, Proietti, \& Terzi, 2015), the directional behavior of a wind turbine is analyzed through SCADA data to build connection between the alignments of wind turbines and performance deviations.

Since SOM based failure detection and prognostic and health management have been widely researched, previous works should be addressed here. In (Lamedica, Prudenzi, Sforna, Caciotta, \& Cencellli, 1996), a short term anomalous load periods prediction technique is proposed. SOM is used for historical loads data classification. In (Hoglund, Hatonen, \& Sorvari, 2000), a computer-host based anomaly detection system is developed while SOM is used to learn the normal behavior from a set of features describing the object. With similar idea, authors in (Fabio A \& Dipankar, 2003) and (Depren, Topallar, Anarim, \& Ciliz, 2005) develop failure and intrusion detection system for different applications. Moreover, in (Tian, Azarian, \& Pecht, 2014), k-nearest neighbor (KNN) algorithm is used to improve SOM for failure detection. SOM can also be used for developing monitoring systems. In (Rigamonti, Zio, Alessi, Astigarraga, \& Galarza, 2015), a monitoring system for operating insulated bipolar transistors is proposed based on SOM. While in (Zhong, Wang, Wu, Zhou, \& Jin, 2016), a SOM based monitoring system is developed solve the visualization monitoring and fault diagnosis problem in chemical industry process. Hence, the general idea that using SOM to capture the features of the normal behavior of an object is powerful in anomaly detection. And this idea is also suit to data driven approach based wind turbine anomaly detection, as failure data is usually not sufficient enough for researchers to catch the failure patterns.

Considering SOM based wind turbine anomaly detection, many works have been published. In (Zhao, Siegel, Lee, \& $\mathrm{Su}, 2013)$, condition monitoring system (CMS) data and SCADA data are used for developing a component level degradation assessment and fault localization framework. However, as is mentioned above CMS is not always available for some wind turbines and it also means more investment for installing devices to get the data. In (Wilkinson, Darnell, Delft, \& Harman, 2014), a comparison study is conducted among NN, SOM and physical model based wind turbine condition monitoring with SCADA data. However, it fails to define the abnormal conditions after the deviation is calculated. Moreover, in (Chen, 2014), the author provides a comprehensive study on wind turbine monitoring using SCADA data. However, SOM is only used as classification tool to distinguish operation states. Experts are required to interpret the results.

Inspired by the previous works, we try to make small steps forward in this field. The main difference of this work is that it proposed a top-down method which is capable of detecting system level anomaly and locating the rooted subcomponent. In this work, self-organizing map (SOM) is adopted to model the normal behavior of a wind turbine by 
projecting high-dimensional variables from SCADA dataset into a two-dimensional space. After that, indicator of system level anomalies is created by calculating the deviations between observed value and normal value. Afterwards, a root subcomponents identification method is developed based on system level deviations. The second difference is that the proposed method only uses SCADA as data source. This brings challenges for quantifying anomalies based on deviation signal. To solve this problem, several filters are designed in this work. Moreover, parameter selection is investigated by using both Pearson correlation coefficient and Kernel canonical correlation coefficient (KCCA) and results are interpreted. The procedure of the developed approach is presented as Figure 2.

The rest of this paper is organized in the following way. In section 2, the approach for anomaly detection and root subcomponent identification are developed. The parameter selection methods are also introduced in this section. In section 3, the dataset adopted in this work is described and cleaned. Moreover, the procedure of SOM construction is discussed. In section 4 , the results of parameter selection are compared, differences are pointed out and a parameter list for normal behavior modeling is proposed. The results of system level anomalies and the root subcomponents are presented and discussed. After that, the conclusions are drawn in section 5 .

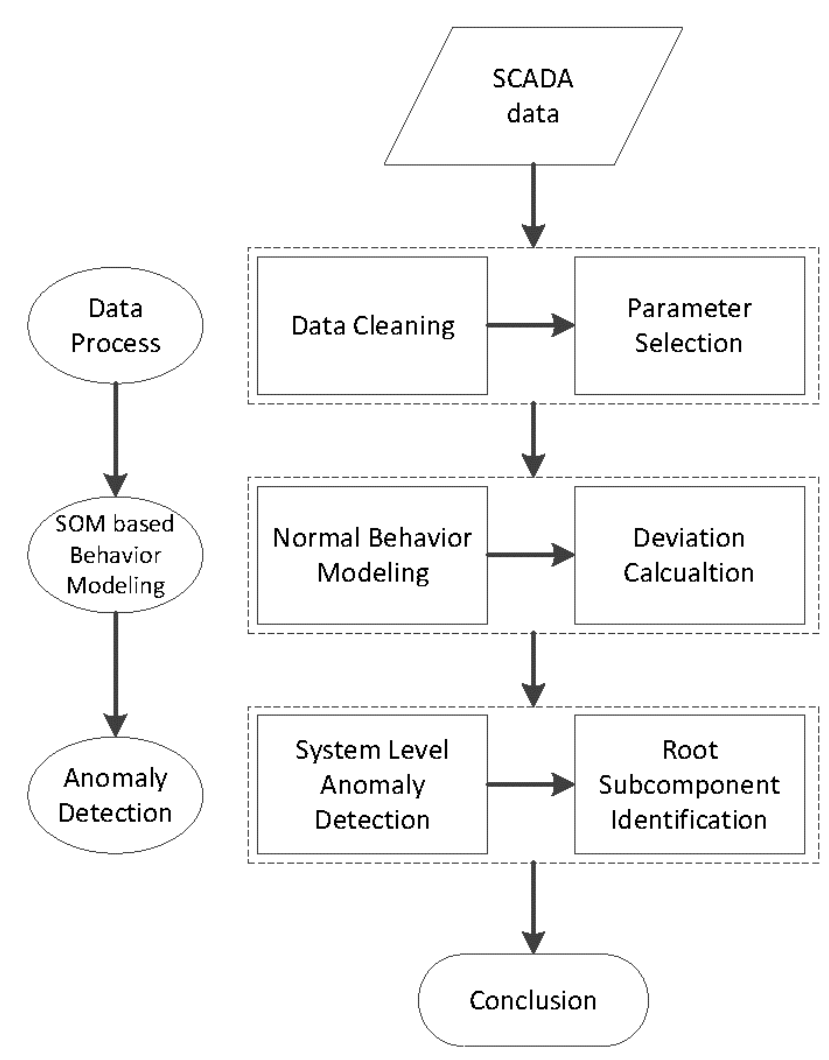

Figure 2. Procedure of the proposed method

\section{APPROACH}

The proposed approach is described in this section. Two methods for parameter selection are investigated, the background knowledge of SOM is introduced, and the indicators for anomaly detection and root subcomponent identification are defined in the following subsections.

\subsection{Parameter Selection}

Parameter selection is very important for modeling normal behavior of a wind turbine through NN (Lapira, Edzel, \& al, 2012). And this problem is often solved by using physical knowledge that which parameter has impact on output power of a wind turbine. However, considering the differences of each dataset, physical knowledge alone is too general to provide a parameter list. Hence, statistical relationship between parameters can be considered as an auxiliary method for parameter selection. In this subsection, after using P-value analysis for general relationship test, both Pearson's correlation coefficient analysis (PCC) and Kernel canonical correlation analysis (KCCA) are adopted for detail investigation.

\subsubsection{P-value analysis}

Null Hypothesis refers to a general statement or default position that there is no relationship between two measured phenomena, or no difference among groups and P-value is widely used as a tool for whether to reject Null Hypothesis (Anderson, Burnham, \& Thompson, 2000).

Based on the definition of $\mathrm{p}$-value, it can be described as follows.

$$
P_{r}(\text { Reject } H \mid H)=P_{r}(p \leq \alpha)=\alpha
$$

$\alpha$ is often pre-fixed as 0.05 (Anderson, Burnham, \& Thompson, 2000), which means if p-value is less than 0.05 , the evidence is strong enough to reject null hypothesis. After using P-value to filter unrelated parameters, correlation coefficient analysis is adopted for next step investigation.

\subsubsection{Pearson's correlation coefficient analysis}

Pearson's correlation coefficients (PCC) between each parameter are calculated with Eq. (2). PCC ranges from -1 to 1 , which represents the extent that two variables are linearly related. As the rank of results is more preferable in this paper, an absolute value of PCC is adopted.

$\left|\rho_{X, Y}\right|=\left|\frac{E(X Y)-E(X) E(Y)}{\sqrt{\left(X^{2}\right)-(E(X))^{2}} \sqrt{E\left(Y^{2}\right)-(E(Y))^{2}}}\right|$

Since PCC is only capable to detect the linear relation between two variables, the results generate by PCC are not accurate enough for parameter selection because most of the variables are associated nonlinearly. Hence, a nonlinear 
relation method, Kernel canonical correlation analysis (KCCA) is also included in this work to compare with PCC.

\subsubsection{Kernel canonical correlation analysis}

KCCA is based on Canonical correlation analysis (CCA), but use Kernels which are functions to map the data into a higher-dimensional feature space. In this manner, it overcomes the drawback that CCA sometimes fails to extract meaningful description of the data due to the linearity of CCA method (Hardoon, Szedmak, \& ShaweTaylor, 2004).

The Kernel function is defined as

$$
K(v, z)=\langle\varnothing(v) \cdot \emptyset(z)\rangle
$$

in which, $v$ and $z$ are variables and $\varnothing(v)$ and $\emptyset(z)$ represent the vectors of $v$ and $z . " \cdot "$ means inner product.

Consider Xand $Y$ are two parameters from a SCADA dataset, after using Kernel function to map them into a higher-dimension, which can be represented as $X^{\prime}$ and $Y^{\prime}$, the correlation can be calculated in the following way.

$$
\rho=\max _{\alpha \beta} \frac{\alpha^{\prime} X X^{\prime} Y Y^{\prime} \beta}{\sqrt{\alpha^{\prime} X X^{\prime} X X^{\prime} \alpha \cdot \beta^{\prime} Y Y^{\prime} Y Y^{\prime} \beta}}
$$

where $\alpha$ and $\beta$ are the direction parameters. The estimation algorithm of $\rho$ is introduced in (Hardoon, Szedmak, \& Shawe-Taylor, 2004).

\subsection{Wind Turbine Normal Behavior Modeling with SOM}

SOM is a sub variant of neural network with unsupervised learning properties. In this paper, the property that it can project a dataset with high dimensional feature into one or two dimensional space is adopted to catch the patterns of input training data (Kohonen, 1982). Before introducing the procedure of training SOM, definition of Best Match Unit $(B M U)$ must be clarified as it the key point of this paper.

Definition of BMU: a BMU is the neuron whose weight vector has the smallest distance measure from the input data.

Considering $x=\left[x_{1}, x_{2} \ldots, x_{n}\right]^{T}$ as the input vector for training iterations, $x_{i}$ represents the parameters selected from the original dataset. Moreover, $w_{i}$ denotes the weight of each neuron, while $w_{i}=\left[w_{i 1}, w_{i 2}, \ldots, w_{i n}\right] i=1,2, \ldots m$. Dimension of the weight vector equal to the number of the input parameters.

For each training step, the distribution of neurons is updated as follows.

$$
\left\|x-w_{u}\right\|=\min \left\{\left\|x-w_{i}\right\|\right\} \text { for all } i=1,2, \ldots, m
$$

In this equation, $w_{u}$ represents the updated neuron weights for next iteration. And $\|\cdot\|$ is the distance between the input vector and the neuron. After it is figure out, the weights of neighbors around $B M U$ are renewed with Eq. (6).

$$
w_{i}(t+1)=w_{i}(t)+\alpha(t) h_{i, w_{c}}(t)\left(x-w_{i}(t)\right)
$$

Where $w_{i}$ represents the weight and $t$ is the iteration step. $\alpha(t)$ is called learning rate that is similar to the other neural networks. $h_{i, w_{c}}$ is a predetermined neighborhood function which can decide whether the weights of a neuron should be updated.

After the training step, the distribution of the neurons in the two-dimension space is considered as the normal behavior model of a wind turbine, and the distance between the new input and the BMU can be used as an indicator for anomaly detection.

\subsection{Anomalies detection and Root Subcomponent Identification}

In this sub section, the definition of anomaly indicator is clarified, the filter for anomaly detection is developed and the root subcomponent identification method is proposed.

\subsubsection{Indicator of a suspicious anomaly}

Deviation shows the difference between the current status and normal behavior. It can be represented with Euclidean distance between new input data and BMU in a twodimensional space in the following way:

$$
\text { Deviation }=\left\|x-w_{B M U}\right\|
$$

in which $x$ is the vector of new input data. As a deviation signal can be observed when the WT does not function well, it is considered as the indicator of potential anomaly occurred in the system.

While in practical, the anomaly data only take a small portion of the whole data sheet. A threshold is defined with quantile function in order to screen out the suspicious data points. The definition of quantile function can be expressed as follows.

$$
\begin{gathered}
Q(p)=\inf \left\{p \leq F_{X}(x)\right\} \\
F_{X}(x)=\operatorname{Pr}(X \leq x)=p
\end{gathered}
$$

in which, $X$ represents deviation signals. Here, the value $p$ is determined by the distributions of both normal behavior and deviations. After that, whenever a deviation out of this interval is observed, it has a high probability to be an anomaly which needs further investigation.

\subsubsection{Anomaly detection and root subcomponent identification}

Among the suspicious data points generated with the filter in section 2.3.1, some of them should be attributed to the automatic control system embedded in a wind turbine or 
turbulence such as changes in wind direction. Therefore, a filter is created in the following form.

$\emptyset(t)=\left\{\begin{array}{ccc}1, d(t-1)<Q(p) \cap(d(t) \cap(t+1))>Q(p) \\ 0, & \text { else }\end{array}\right.$

where $t$ is the observing time. If the data sequence satisfies Eq. (10), then $\emptyset(t)$ equals to 1 , which means that $t$ is the first time that an anomaly can be detected. While $d(t)$ represent the deviation signal at time $t$. This filter is inspired by patterns of real failure signal suffering a sudden increase from normal value to a relative higher value and sojourn for at least one period.

In order to go deeper into a component level to trace the root cause of a system anomaly, Eq. (7) provides a hint. As each parameter contributes to the deviation, the contribution proportion (CP) can be considered as an index for root component identification. The index is defined as follows.

$$
\mathrm{CP}_{\mathrm{i}}=\frac{\left(\mathrm{v}_{\mathrm{i}}-\mathrm{w}_{\mathrm{i}}^{\mathrm{BMU}}\right)^{2}}{\sum_{\mathrm{i}=1}^{\mathrm{n}}\left(\mathrm{v}_{\mathrm{i}}-\mathrm{w}_{\mathrm{i}}^{\mathrm{BMU}}\right)^{2}} \times 100 \%
$$

in which, $v_{i}$ is the parameter in the new input data, $w_{i}^{B M U}$ is the corresponding weight of the BMU. A subcomponent may suffer an anomaly if the corresponding parameter contributes much more to deviation signals than the others.

\section{APPLICATION}

Considering the specialty of different wind turbine SCADA data, the application of the approach developed in section 2 is represented in this part.

\subsection{Data Processing}

The data points that represent the normal operation conditions should be selected for the normal behavior model. To reach this goal, the wind turbine theoretical power curve is used as reference. Figure 3 shows the reference power curve and Figure 4 represents the power curve generated from the original dataset.

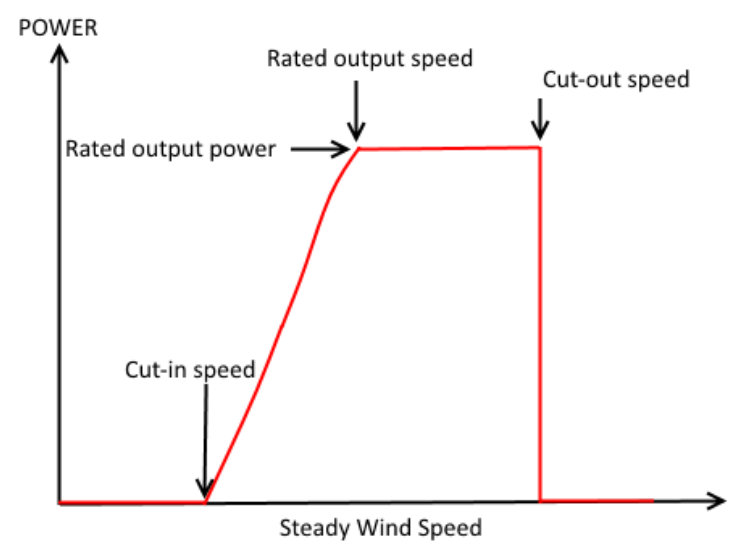

Figure 3. Theoretical power curve of a wind turbine

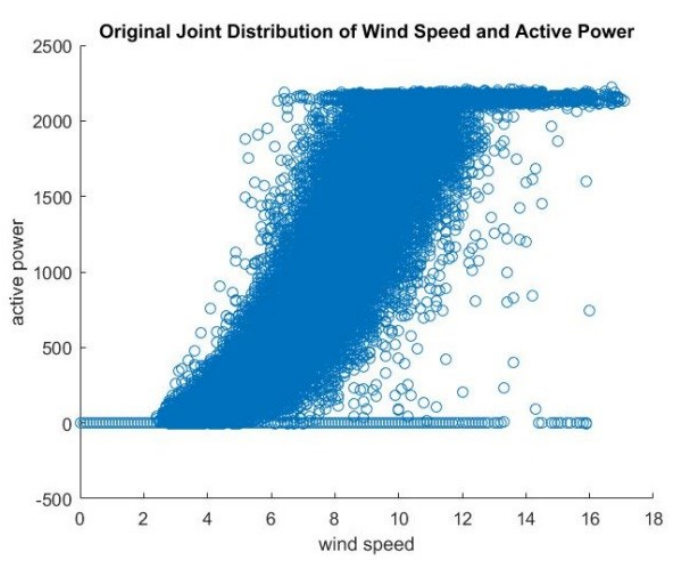

Figure 4. Power curve based on original dataset

Comparing with the theoretical power curve of a WT shown in Figure 3, suspicious data points should be filter out by some criterions. The procedure of data cleaning process is represented in the subsequent Table 1.

Moreover, since units of each parameter are different, when trying to model system level behavior, some of the parameters with small value range cannot have equal chance to impact on the model. Hence, the data should be normalized. Based on the dataset adopted in this paper, each parameter is normalized with the following Eq. (12)

$$
N_{\text {vector }}=\left(V_{i}-\operatorname{Min}_{v}\right) \div\left(\operatorname{Max}_{v}-\operatorname{Min}_{v}\right)
$$

Where $N_{\text {vector }}$ represents the normalized data vector and $V$ means the original data vector.

The original dataset adopted in this work is a real SCADA dataset covering two months operation period of a $2.5 \mathrm{MW}$ wind turbine and the sampling period is every 20 seconds. According to the data reccord, no serious failures are observed. In the original dataset, there are 53 parameters collected from to both wind turbine subcomponents and the power grid it integrated. The size of the original SCADA dataset is $205595 \times 53$. After filtering out the bad data points, the size of the dataset is $204893 \times 53$. The healthy dataset which is used for training SOM contains $170382 \times 53$ after the whole data process. The prepared dataset for normal behavior modeling is show as Figure 5.

\begin{tabular}{l|l}
\hline No. of Steps & Criterion Description \\
\hline 1. & $\begin{array}{l}\text { Data points containing } \\
\text { negative power output. }\end{array}$ \\
\hline 2. & $\begin{array}{l}\text { Data points containing } \\
\text { warning signals. }\end{array}$ \\
\hline 3. & $\begin{array}{l}\text { Data points which are far } \\
\text { from the theoretical curve. }\end{array}$ \\
\hline
\end{tabular}

Table 1. Procedure of data cleaning 


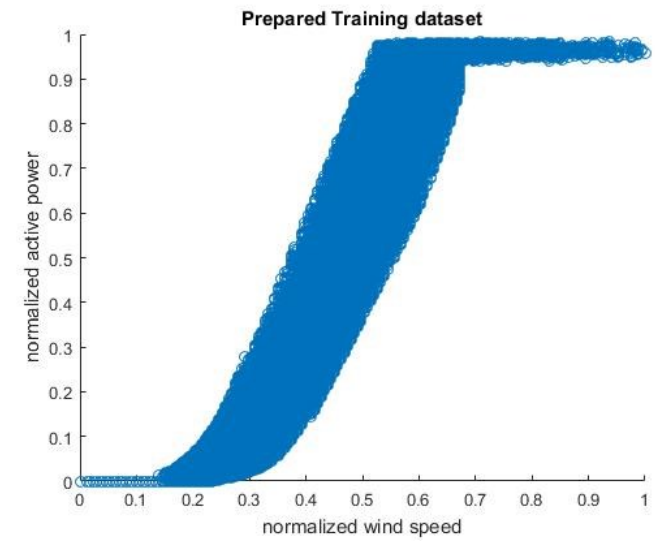

Figure 5. Processed power curve of a wind turbine

\subsection{SOM Construction and normal behavior modeling}

Before modeling normal behavior of a WT, a SOM must be constructed with all the parameters settled. In this part, settings of the SOM are described.

To build a SOM, the number of neurons is very important as it has direct influence on the training results. While less number of neurons will lead to overlap of features in different clusters, too more neurons will attribute to the separation of data points with similar characteristics. Inspired by (Vesanto, Himberg, Alhoniemi, \& Parhankangas, 2000), the number of neurons is determined based the following empirical function.

$$
n=5 \sqrt{v_{\text {train }}}
$$

in which, $v_{\text {train }}$ represents the number of vectors inside the training dataset. The alignment of the neurons is defined as "hextop", while the initialization of weights of each neuron is complete with default setting.

Batch training algorithm is used in this paper. Batch training algorithm is also an iterative algorithm, instead of inputting one vector for each training step; the whole data set is used for training before updating the weights of neurons. For batch training algorithm, based on Eq. (6) weight vectors are updated as:

$$
w_{i}(t+1)=\frac{\sum_{j=1}^{n} h_{i, w_{c}}(t) x_{j}}{\sum_{j=1}^{n} h_{i, w_{c}}(t)}
$$

with neighborhood function $h_{i, w_{c}}(t)$ and learning rate $\alpha(t)$ as default settings.

After data process, the prepared dataset is adopted as input for normal behavior modelling. The training results are shown in Figure 6.
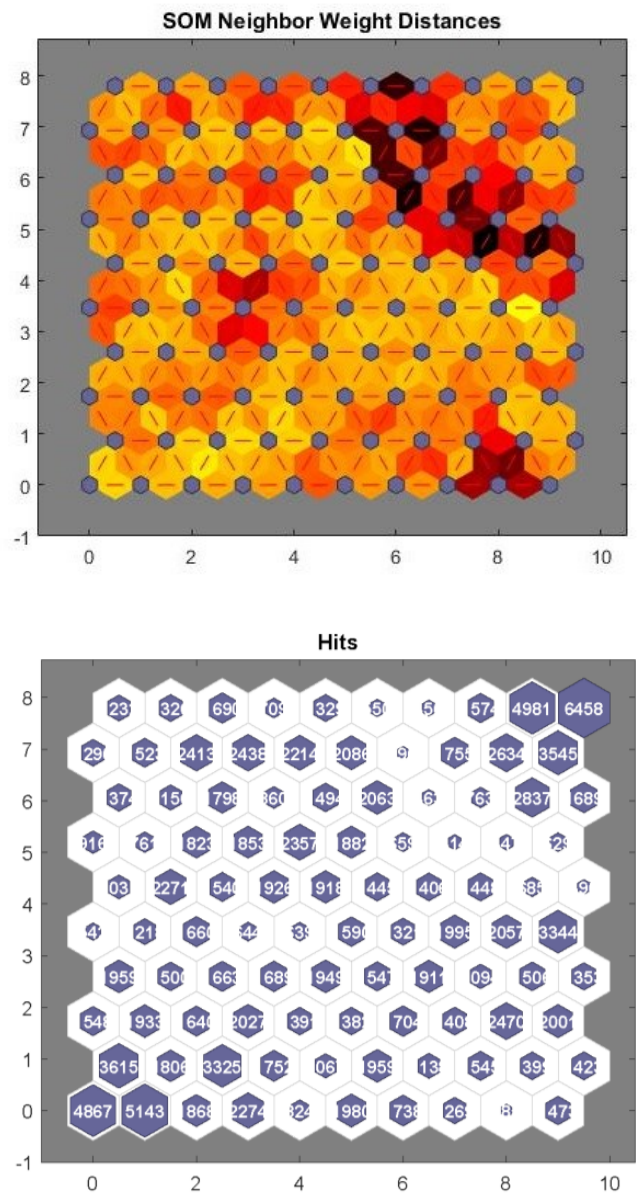

Figure 6. (top) SOM neighbor weight distances and (down) number of data points in each cluster

In Figure 6, the image on top shows the distance between each cluster, darker color means longer distance. The figure in bottom shows the number of data points in each neuron. Based on practical experience, since each neuron contains a certain number of data points, the SOM adopted in this paper functions well. Moreover, the alignment of all neurons after training is shown as Figure 7.
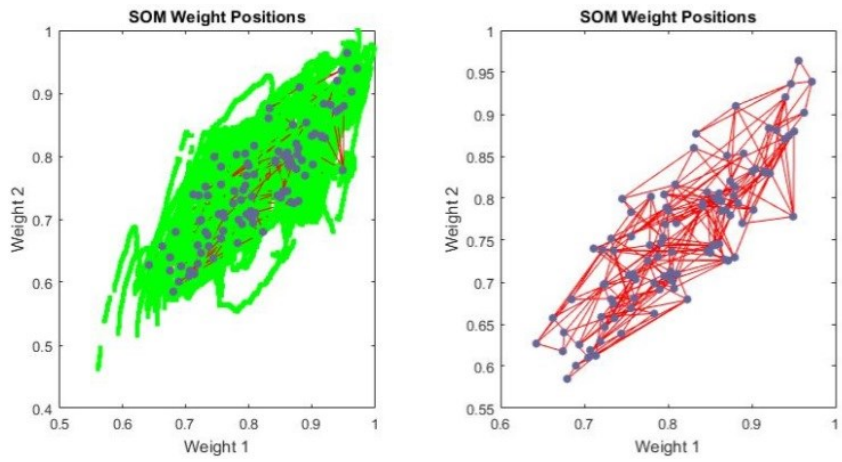

Figure 7. Alignment of neurons after training 
In the left image, the background is the distribution of weight 1 and weight 2 of each neuron and the corresponding parameters are gearbox bearing temperature and gearbox oil temperature. The right image is the distribution of neurons and the red line represents the connections among neurons. Figure 7 shows that all the neurons are distributed homogeneously within the normal area defined by the training dataset.

\subsection{Threshold Setting}

As was mentioned in subsection 2.3.1, to filter out the ordinary deviation signals, a Quantile function is adopted. Based on the results generated by applying SOM to both normal dataset and original dataset, a histogram of both deviation signals and normal behavior distributions is used to determine the quantile value. The histogram is shown in Figure 8. The data points with darker color represent the normal behavior of a wind turbine. Data points in the red circle are screen out for anomaly investigation with the quantile value as $85 \%$. The corresponding deviation is 0.10932 , which means that any deviation signal larger than 0.10932 is suspicious.

\section{RESULTS DISCUSSIONS}

First, the results of parameter selection are discussed in this part. Besides, the results of anomaly detection and sub component identification are also represented in this part.

\subsection{General relationship test results}

As is mentioned in 2.1.1, P-value analysis is adopted to test are these parameters from SCADA dataset related to each other from a general way. Any result that is larger than 0.05 means that the target two parameters are not related statistically. Since in this paper, we focus on the relationship between WT power output and other parameters, only related results are discussed here.

Based on the physical knowledge, since all the components are interconnected, they should be related to each other by some extent. The results prove this assumption as most of $\mathrm{P}$ values between WT power output and other parameters are zero. While in Table 2 there are some results not equal to zero, the values are still far less than 0.05 to accept the Null Hypothesis.

\begin{tabular}{l|l}
\hline Parameter & P-value \\
\hline wind direction & $4.38 \mathrm{e}-141$ \\
\hline power factor & $1 ., 84 \mathrm{e}-48$ \\
\hline nacelle temperature & 0.0165 \\
\hline ambient temperature & $7.47 \mathrm{e}-47$ \\
\hline reactive power & $1.49 \mathrm{e}-15$ \\
\hline yaw & $1.66 \mathrm{e}-06$ \\
\hline
\end{tabular}

Table 2. P-value test results

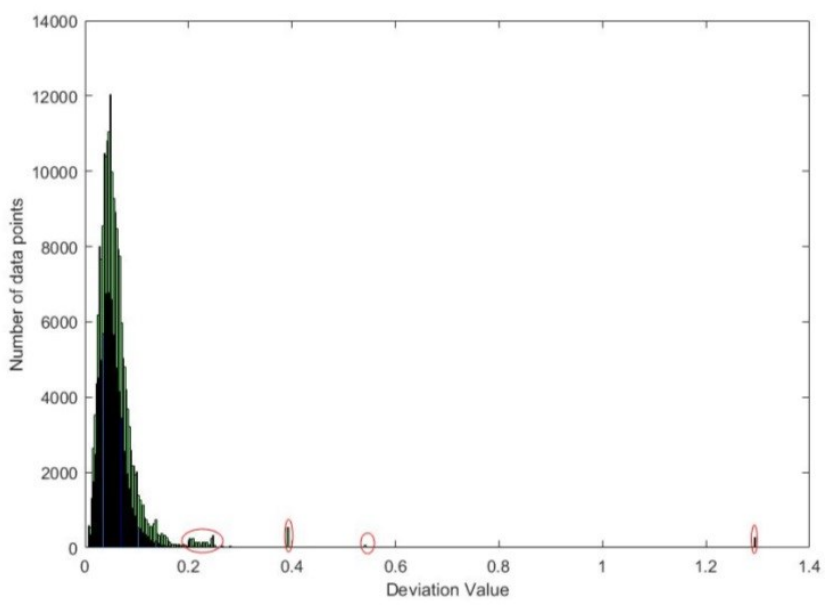

Figure 8. Histogram of deviation signals and normal distributions for threshold setting

\subsection{Parameter List Suggestion}

Since the method is created for wind turbine anomaly detection, all the measurements related to the transmission grid which the wind farm integrated are filtered out. According to (Sun, Li, Wang, \& Lei, 2016) (Lapira, Edzel, $\&$ al, 2012), parameters that have impact on output power should be selected based on practical knowledge from the physical connections among the sub components. Inspired by this idea, correlation coefficient analysis between Active_Power and other parameters is conducted. Table 3 shows the results from PCC and KCCA.

In this table, both PCC and KCCA can detection the statistical relation between active_power and other parameters. All the parameters which are considered to have impact on wind turbine power output based on physical knowledge are all included in Table 3. The differences between two results are the ranks of three pitch angle related parameters. Since pitch angle has significant impact on power output, KCCA based rank is more reasonable since KCCA is capable of detecting nonlinear relation between variables. However, both these two methods show low ranks of yaw, ambient temperature and nacelle temperature while last two parameters are often selected for modeling wind turbines behavior. The reason is that the measurements of these three parameters are almost constant and do not change frequently, while both PCC and KCCA are sensitive to parameters distributed in a wide range.

Combined with the practical knowledge in this domain and inspired by (Lapira, Edzel, \& al, 2012), six parameters are selected from the SCADA data (i.e., gearbox bearing temperature, gearbox oil temperature, nacelle temperature, rotor speed, generator bearing temperature and pitch location 2). 


\begin{tabular}{|c|c|c|}
\hline Rank & PCC & KCCA \\
\hline 1. & generator torque & generator torque \\
\hline 2. & wind speed & wind speed \\
\hline 3. & generator $\mathrm{u} 1$ temperature & generator speed \\
\hline 4. & generator $\mathrm{v} 1$ temperature & rotor speed \\
\hline 5. & generator w 1 temperature & gearbox temperature 1 \\
\hline 6. & gearbox temperature 1 & gearbox temperature 2 \\
\hline 7. & gearbox temperature 2 & pitch location 2 \\
\hline 8. & generator speed & pitch location 1 \\
\hline 9. & rotor speed & pitch location 3 \\
\hline 10. & converter temperature & generator $\mathrm{u} 1$ temperature \\
\hline 11. & gearbox oil temperature & generator $\mathrm{v} 1$ temperature \\
\hline 12. & $\begin{array}{l}\text { generator bearing } \\
\text { temperature } 2\end{array}$ & generator w 1 temperature \\
\hline 13. & $\begin{array}{c}\text { gearbox entrance } \\
\text { temperature }\end{array}$ & converter temperature \\
\hline 14. & $\begin{array}{l}\text { gearbox bearing } \\
\text { temperature }\end{array}$ & gearbox oil temperature \\
\hline 15. & gearbox oil pressure & $\begin{array}{c}\text { gearbox entrance } \\
\text { temperature }\end{array}$ \\
\hline 16. & pitch location 3 & $\begin{array}{l}\text { generator bearing } \\
\text { temperature } 2\end{array}$ \\
\hline 17. & pitch location 1 & $\begin{array}{l}\text { gearbox bearing } \\
\text { temperature }\end{array}$ \\
\hline 18. & pitch location 2 & gearbox oil pressure \\
\hline 19. & wind direction & wind direction \\
\hline 20. & ambient temperature & yaw \\
\hline 21. & yaw & nacelle temperature \\
\hline 22. & nacelle temperature & ambient temperature \\
\hline
\end{tabular}

Table 3. Results of Correlation coefficient analysis

\subsection{Anomalies Detection and Root Subcomponents Identification}

First, results in Figure 8 should be discussed. This figure shows that there are some extreme deviation values which are more than 1.2 and far from the main distribution. Based on the definition of anomaly in 2.3, they could be real anomalies which have significant impact on WT power output; however, when checking the original dataset for these cases, all these extreme values should be attributed to the sensors for measuring rotor speed in low wind scenarios. In this case, these data points are filtered out. Also from the patterns shown in the deviation signal, these extreme values

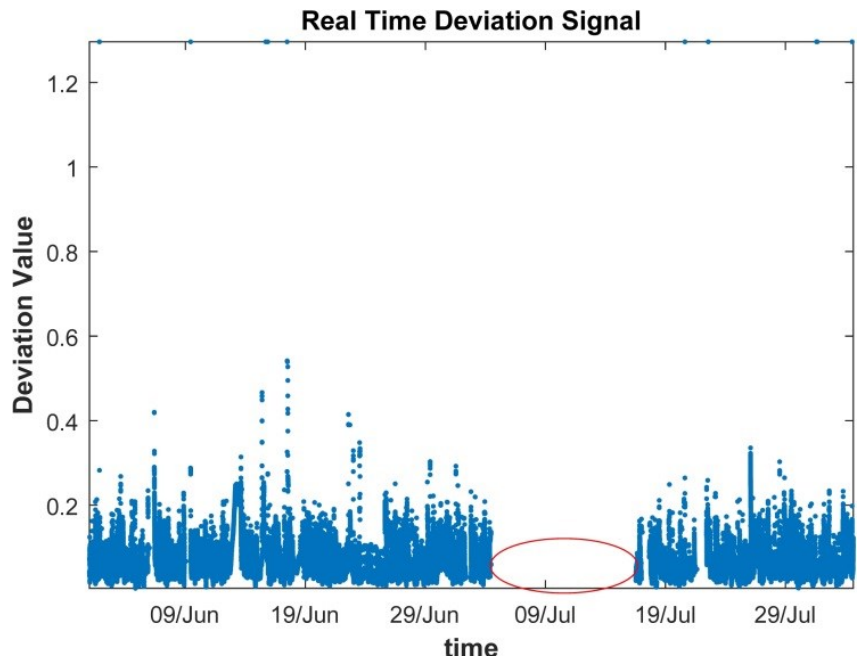

Figure 9. Real time deviation signals in two months

can be excluded from anomaly detection. Details will be discussed in 4.3.1, case 2 .

Figure 9 shows deviation signals in two months without applying the quantile filter. In this figure, significant jumps are observed while most of the deviations lay in the normal zone. The long gap in red ellipse shown in Figure 9 is due to the longest shutdown time in two months.

For system level anomaly detection, the method proposed in this paper is verified using warning signals from SCADA dataset as reference. For warning signal which is also included in SCADA dataset, has binary value 0 and 1 . Since this parameter is not directly related to failure or anomaly, it is often considered as a reminder that the condition of the wind turbine is working poorly. Hence, we assume that major anomalies and potential failures are hidden in these cases. What should be mentioned is that according to the SCADA data set, the warning signal first-hitting-time happened 370 times in two months. Although most of them are not for anomalies, they can be used to test the deviation signals after applied the filters as Eqs. (8), (9), (10). In the following subsection, several cases are selected to prove that the proposed anomaly detection method is effective as all these deviation signals can track the warning signal precisely.

\subsubsection{Case study (model verification):}

To verify the anomaly detection method proposed in 2.3, warning signal is adopted as reference. According to the original data, the first time of the warning signal hits one is located and the sojourn time that the warning signal stays in one is calculated. Several cases are selected to test the method. In the following figures, the points in red ellipse are corresponding to the first time when warning signals hit one. 

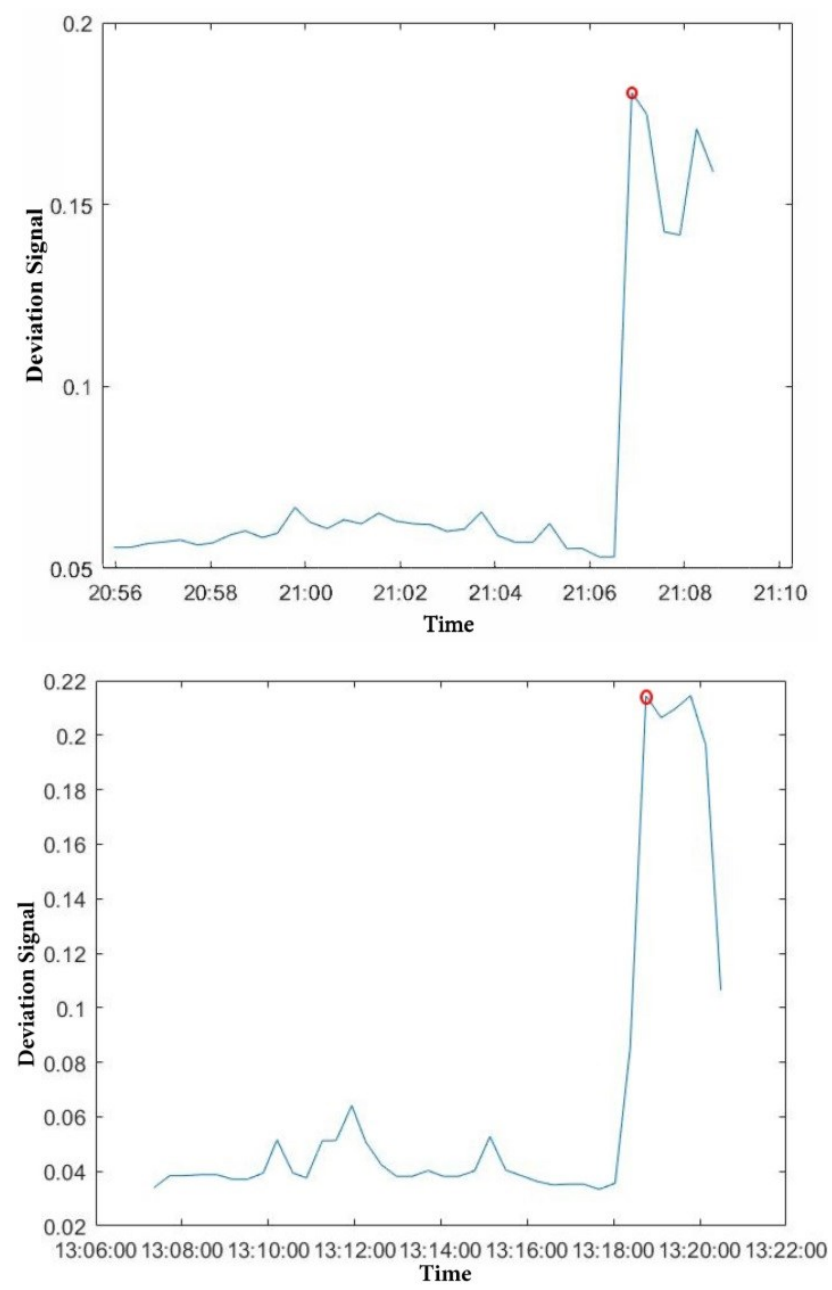

Figure 10. Case 1 WT keeps working when warning signal hit one

Case 1, wind turbine keeps working when warning signal hits one. As is shown in Figure 10, the proposed method tracks the warning signal properly, and can find sharp increase several periods before the warning signal.

Case 2, from the results generated by SOM, the deviations from the "normal" behaviors show that some 'big' jumps exist and this should be investigated. As is shown in Figure 11, when 'big' jumps occur, all the sub parameters show exactly same patterns, i.e. a sudden increase around the warning time. In practical, multi-failure at the same time is almost impossible and the ratio of each parameter count for the deviation does not change in this case. What is more, when check original data, it is almost sure that this 'big jump' case should be attributed to sensor errors.

Case 3 is corresponding to the longest gap mentioned 4.3 which represents a long time shut down for this WT which is nearly one day. In this case, there may be a real failure which needs to be fixed or it is just a scheduled maintenance.
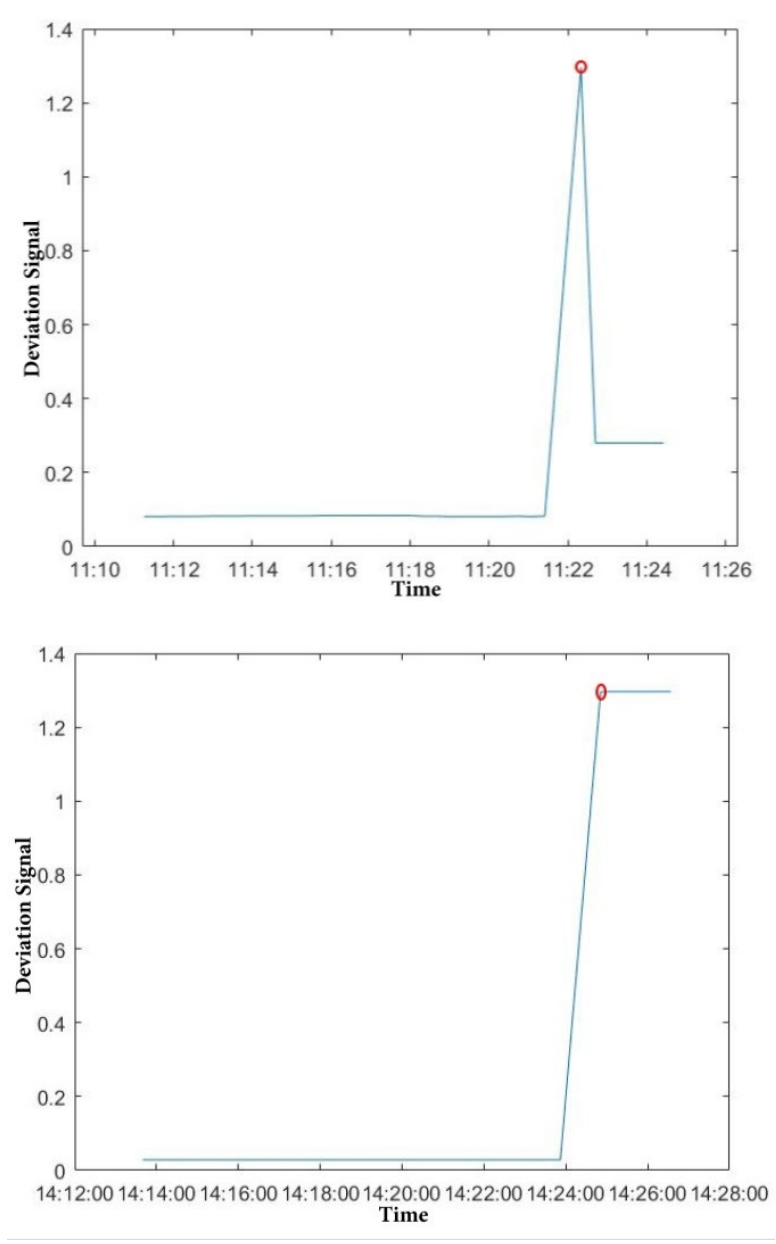

Figure 11. Case 2 extreme cases in which deviation values are too big.

From Figure 12, the deviation signal during this time scale suddenly increased and suffered vibration in the next several periods. Hence, there may be an anomaly among the sub conponents which need further investigation.

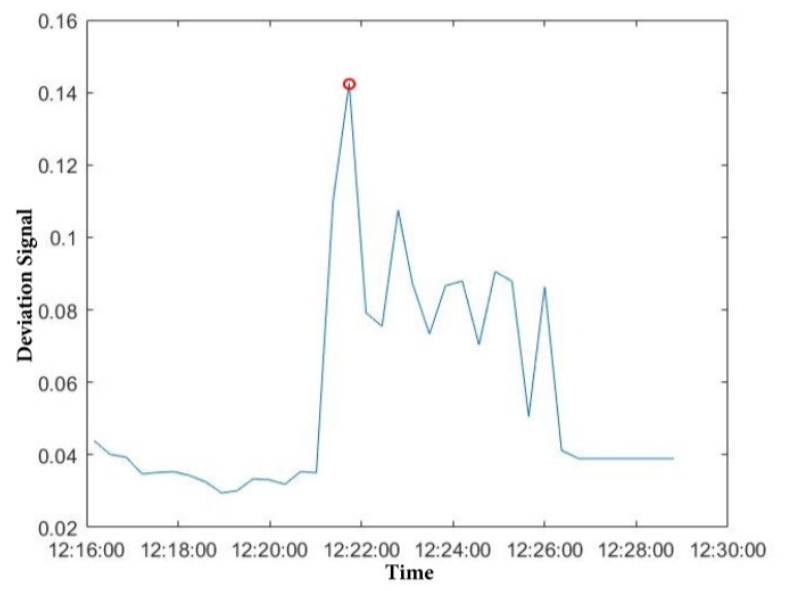

Figure 12. Case 3 the longest shut down time in two months 

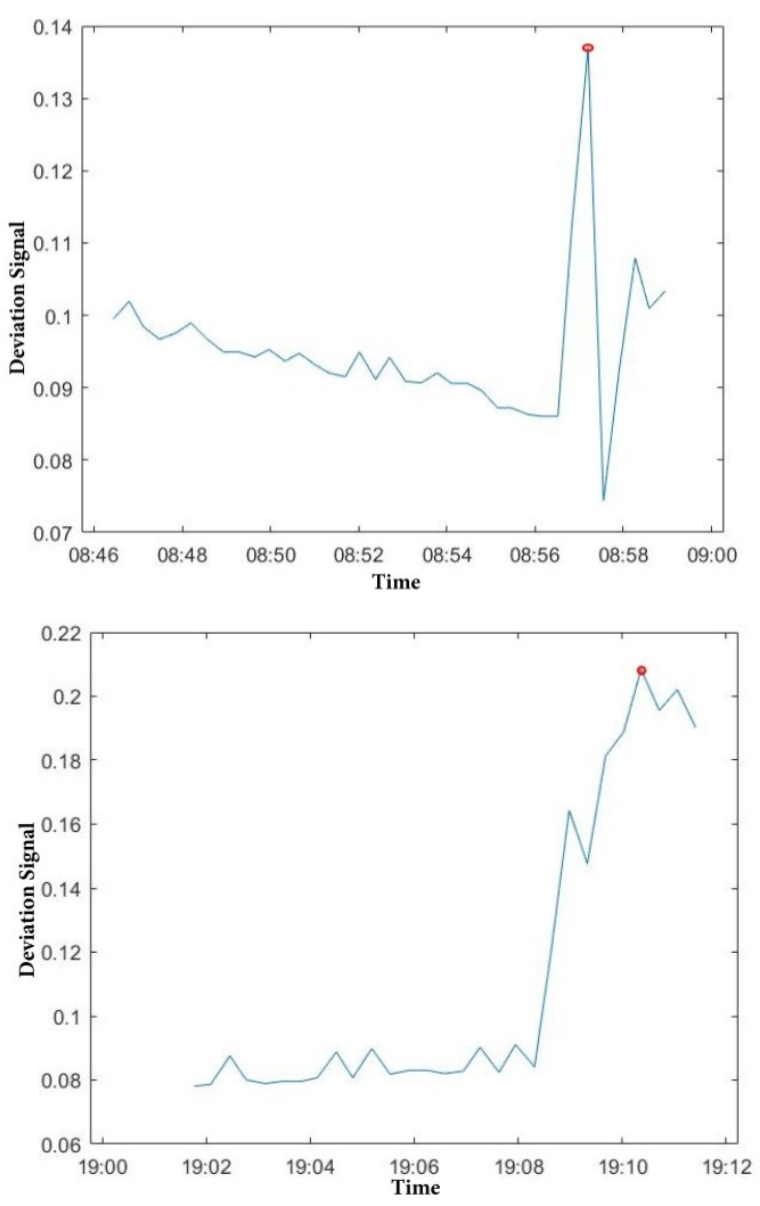

Figure 13. Case 4 WT is shut down after warning signal hits one

Case 4 , is when the value of fault signal hit 1 , WT is not working. In Figure 13, the patterns of the curves do not show significant differences from case 1 . However, when checking the average value of deviation signals, the average level in case 4 is higher, which implies that the condition of the wind turbine is worse than in case 1 .

Based on the proposed method, only 5 cases are confirmed as anomalies after checking the original SCADA data. They are shown in Figure 10, 12, 13. The SOM based anomaly detection method is sensitive to the changes of the parameters. This lead to the cases shown in Figure 11, as sensor errors can bring interferences. Also for failures caused by ageing, which often has a relatively long time process, the remaining life model based failure detection method is more powerful. But whenever an anomaly occurs and the corresponding parameters change, the proposed method can detect it.

For root sub component identification, Figure 14 is provided for discussion. The time scale in this case is about $10 \mathrm{mins}$, the time point in the figure is when an anomaly is detected.
The red line represents the average contribution of each parameter.

In this case, the largest part of contribution is attributed to both gearbox bearing temperature and gearbox oil temperature. When looking at the beginning of this period, there is a sudden increase in the pitch angle's contribution to the total deviation. After that, increment cause by rotor speed is observed, and a lag for the increment of gearbox bearing temperature and generator bearing temperature are also appeared in this figure. This case indicates that pitch system suffered a turbulence cause by the change of wind direction in during operation and gearbox bearing and oil need inspection in detail.

Another case for sub component identification is shown as Figure 15. In this case, at the beginning of this period, the contribution ratio of rotor speed witnessed turbulence due to a sudden change of wind speed. However, for almost the whole period, nacelle temperature contributed much more than the average level while other parameters stayed lower than the average level. Hence, a conclusion can be made that some other components may suffer an anomaly which caused the increase in the contribution ratio of nacelle temperature.

After discussing all the results by different cases, the proposed anomaly detection method can find out system anomalies lurking in the sub components properly. And the root subcomponents identification method can provide suggestions on which sub component needs further inspection.

\section{CONCLUSION}

In this work, a data driven method for Wind Turbine system level anomaly detection and root sub-component identification is developed. Wind turbine SCADA data is adopted and several parameters are selected based on physic knowledge and correlation coefficient analysis for normal behavior modeling. This model which is based on SOM projects higher-dimensional SCADA data into a twodimension-map. Afterwards, the Euclidean distance based indicator for system level anomalies is defined and a filter is created to screen out suspicious data points based on quantile function. Moreover, a failure data pattern based criterion is created for anomaly detection from system level. In order to track which sub-component should be responsible for an anomaly, a contribution proportion (CP) index is proposed. For parameter selection, the results shows that both PCC and KCCA can help determining which parameter is suitable for building model, but tracking the changes within a data set is not appropriate for every case. Besides, the results of system anomalies prove the efficiency of the proposed method and the CP index is effective for figuring out which sub-component is responsible for the anomaly. The future works are in two directions: 

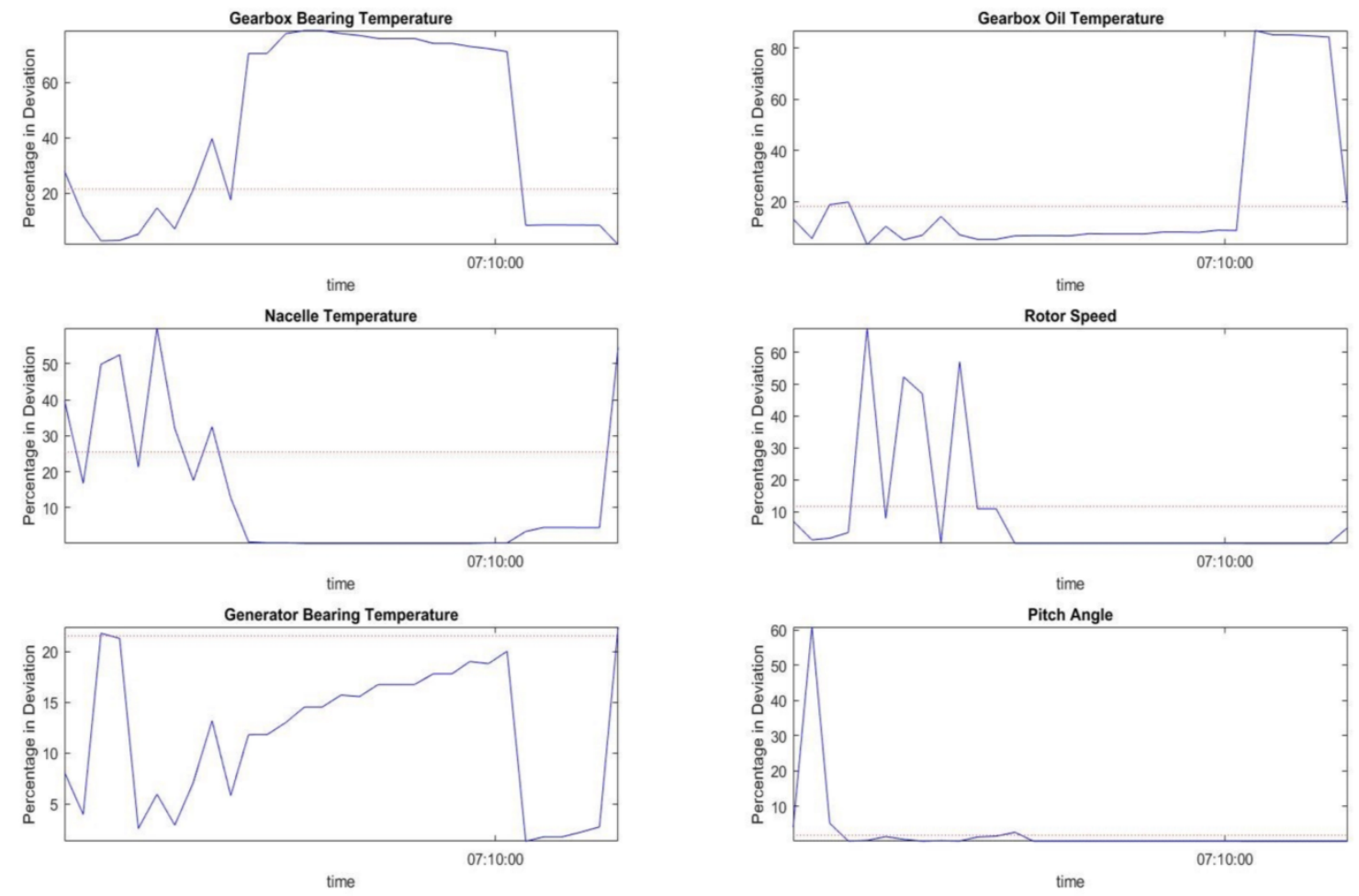

Figure 14. Root sub component identification case study (abnormal Gearbox)
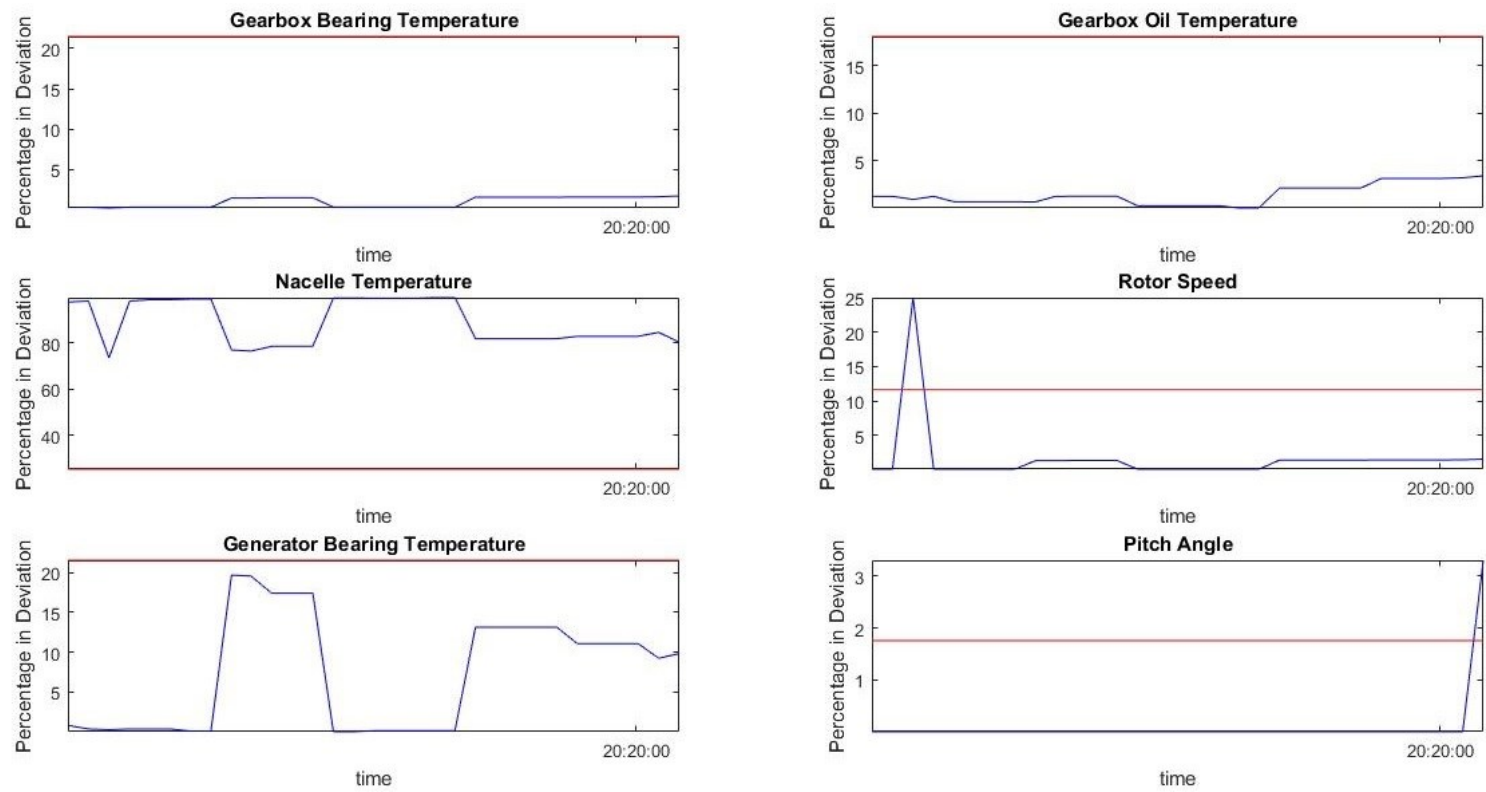

Figure 15. Root sub component identification case study (abnormal Nacelle temperature)

1. Parameter selection for data driven modeling needs more investigation. An approach without an assumption on the relationship between variables is preferred.

2. The cumulative deviation curve shown in Figure 16 can be considered as wind turbine performance degradation from a long term perspective because it shows how the condition of a wind turbine changes in a period. According to (Snchez-Silva, 2015), degradation can be considered as the decrease in capacity of an engineered system over time, as measured by one or more performance indicators. Figure 16 represents the cumulative deviation which is the performance indicator as it shows a successive process that the condition of a wind turbine is getting worse. This curve can be used for remaining useful life estimation and 
performance \& maintenance evaluation which need further investigation.

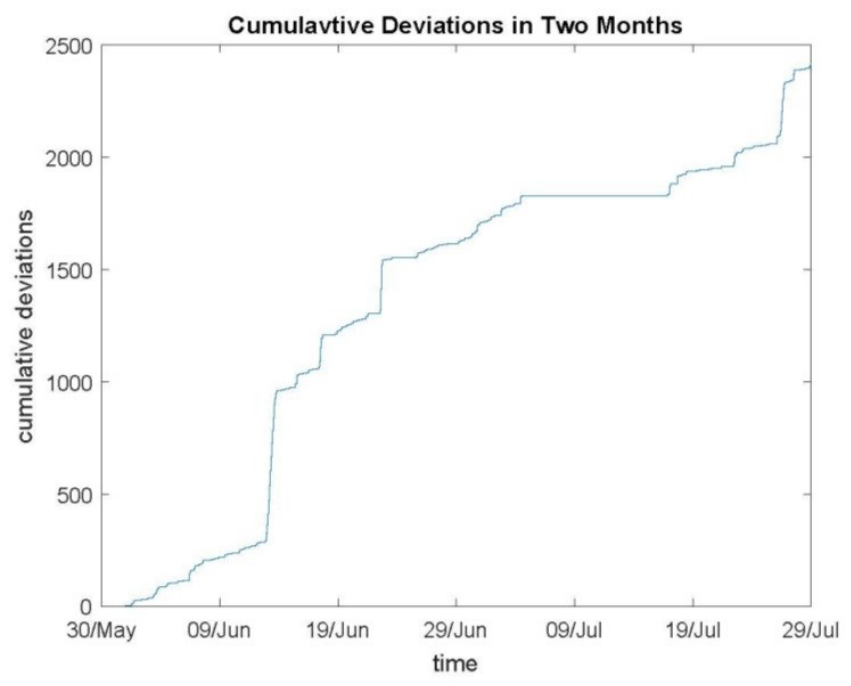

Figure 16. Cumulative deviations in two months

\section{REFERENCES}

Anderson, D. R., Burnham, K. P., \& Thompson, W. L. (2000). Null hypothesis testing: problems, prevalence, and an alternative. The journal of wildlife management, 912-923.

Breteler, D., Kaidis, C., Tinga, T., \& Loendersloot, R. (2015). Physics based methodology for wind turbine failure detection, diagnostics \& prognostics. In EWEA 2015 (pp. 1-9). European Wind Energy Association.

Bruce, T., Long, H., \& Dwyer-Joyce, R. S. (2015). Dynamic modelling of wind turbine gearbox bearing loading during transient events. Renewable Power Generation, IET, 9(7), 821-830.

Castellani, F., Astolfi, D., Sdringola, P., Proietti, S., \& Terzi, L. (2015). Analyzing wind turbine directional behavior: SCADA data mining techniques for efficiency and power assessment. Applied Energy.

Chen, B. (2014). Automated On-line Fault Prognosis for Wind Turbine Monitoring using SCADA data. Durham University.

de Andrade Vieira, R., \& Sanz-Bobi, M. (2013). Failure Risk Indicators for a Maintenance Model Based on Observable Life of Industrial Components With an Application to Wind Turbines. IEEE Transactions on Reliability, 62(3), 569 - 582.

de Azevedo, H. D., Araújo, A. M., \& Bouchonneau, N. (2016). A review of wind turbine bearing condition monitoring: State of the art and challenges. Renewable and Sustainable Energy Reviews, 56, 368-379. de Bessa, I. V., Palhares, R. M., D'Angelo, M. F., \& Chaves Filho, J. E. (n.d.). Data-driven fault detection and isolation scheme for a wind turbine benchmark.

Depren, O., Topallar, M., Anarim, E., \& Ciliz, M. K. (2005). An intelligent intrusion detection system (IDS) for anomaly and misuse detection in computer networks. Expert systems with Applications, 713722.

European Wind Energy Association. (2016). Wind in power 2015 European statistics.

Fabio A, G., \& Dipankar, D. (2003). Anomaly Detection Using Real-Valued Negative Selection. Genetic Programming and Evolvable Machines, 383-403.

Hardoon, D. R., Szedmak, S., \& Shawe-Taylor, J. (2004). Canonical correlation analysis: An overview with application to learning methods. Neural computation, 16(12), 2639-2664.

Hoglund, A. J., Hatonen, K., \& Sorvari, A. S. (2000). A computer host-based user anomaly detection system using the self-organizing map. In Neural Networks, 2000. IJCNN 2000, Proceedings of the IEEE-INNS-ENNS International Joint Conference on (pp. 411-416).

Kandukuri, S. T., Karimi, H. R., \& Robbersmyr, K. G. (2016). A review of diagnostics and prognostics of low-speed machinery towards wind turbine farmlevel health management. Renewable and Sustainable Energy Reviews, 53, 697-708.

Kohonen, T. (1982). Self-organized formation of topologically correct feature maps. Biological cybernetics, 59-69.

Lamedica, R., Prudenzi, A., Sforna, M., Caciotta, M., \& Cencellli, V. O. (1996). A neural network based technique for short-term forecasting of anomalous load periods. IEEE Transactions on Power Systems, 1749-1756.

Lapira, Edzel, \& al, e. (2012). Wind turbine performance assessment using multi-regime modeling approach. Renewable Energy(45), 86-95.

Marhadi, K. S., \& Skrimpas, G. A. (2015). Automatic Threshold Setting and Its Uncertainty Quantification in Wind Turbine Condition Monitoring System. International Journal of Prognostics and Health Management,6(Special Issue Uncertainty in PHM).

Milborrow, D. (2006). Operation and maintenance costs compared and revealed. Windstats Newsletter, 19, 1-3.

Park, B., Sohn, H., Malinowski, P., \& Ostachowicz, W. (2016). Delamination localization in wind turbine blades based on adaptive time-of-flight analysis of noncontact laser ultrasonic signals. Nondestructive Testing and Evaluation, 1-20.

Rigamonti, M. a., Zio, E., Alessi, A., Astigarraga, D., \& Galarza, A. (2015). A Self-Organizing Map-Based Monitoring System for Insulated Gate Bipolar 
Transistors Operating in Fully Electric Vehicle. Annual Conference of the Prognostic and Health Management Society 2015.

Santos, P., Villa, L. F., Reñones, A., Bustillo, A., \& Maudes, J. (2015). An SVM-based solution for fault detection in wind turbines. Sensors, 15(3), 56275648.

Schlechtingen, M., Santos, I. F., \& Achiche, S. (2013). Wind turbine condition monitoring based on SCADA data using normal behavior models. Part 1: System description. Applied Soft Computing, 13(1), 259-270.

Snchez-Silva, M. (2015). Degradation: Data Analysis and Analytical Modeling. In Reliability and Life-Cycle Analysis of Deteriorating System (pp. 79-82). Springer.

Sun, P., Li, J., Wang, C., \& Lei, X. (2016). A generalized model for wind turbine anomaly identification based on SCADA data. Applied Energy, 168, 550567.

Tian, J., Azarian, M. H., \& Pecht, M. (2014). Anomaly Detection Using Self-Organizing Maps-Based KNearest Neighbor Algorithm. Proceedings of the European Conference of the Prognostics and Health Management Society. Citeseer.

Vesanto, J., Himberg, J., Alhoniemi, E., \& Parhankangas, J. (2000). SOM toolbox for Matlab 5. Helsinki, Finland: Helsinki University of Technology.

Wilkinson, M., Darnell, B., Delft, T. V., \& Harman, K. (2014). Comparison of methods for wind turbine condition monitoring with SCADA data. IET Renewable Power Generation, 390-397.

Xiang, J., Watson, S., \& Liu, Y. (2009). Smart monitoring of wind turbines using neural networks. In Sustainability in Energy and Buildings (pp. 1-8). Springer.

Yang, D., Li, H., Hu, Y., Zhao, J., Xiao, H., \& Lan, Y. (2016). Vibration condition monitoring system for wind turbine bearings based on noise suppression with multi-point data fusion. Renewable Energy, 92, 104-116.

Zhao, W., Siegel, D., Lee, J., \& Su, L. (2013). An integrated framework of drivetrain degradation assessment and fault localization for offshore wind turbines. IJPHM Special Issue on Wind Turbine PHM (Color), 46-58.

Zhong, B., Wang, J., Wu, H., Zhou, J., \& Jin, Q. (2016). SOM-based visualization monitoring and fault diagnosis for chemical process. 2016 Chinese Control and Decision Conference (CCDC), (pp. 5844-5849). 